\title{
Hepatite C em Profissionais da Saúde: Risco de Exposição e Infecção
}

Luiz Alberto de Souza Ciorlia'

Dirce Maria Trevisan Zanetta²

\author{
Hepatitis C in Healthcare Workers: \\ Risk of Exposure and Infection
}

'Médico do Trabalho, Coordenador do Serviço de Medicina Ocupacional da Faculdade de Medicina de São José do Rio Preto (SP);

${ }^{2}$ Chefe do Departamento de Epidemiologia e Saúde Coletiva da Faculdade de Medicina de São José do Rio Preto (SP).
Este artigo de revisão discute aspectos da hepatite $C$ em profissionais da saúde e seu risco de exposição e infecção. Foram abordados temas como associação entre patógenos veiculados ao sangue e profissionais da saúde, hepatite C (epidemiologia, diagnóstico e prevenção), comunicação e registro de acidentes do trabalho com material biológico (sangue e fluidos corpóreos), e relação entre extensão e conseqüências dos acidentes com objetos perfurocortantes e exposição mucocutânea e a hepatite $C$.

Palavras-chaves Hepatite C, Profissionais da Saúde, Acidente de Trabalho, Material Biológico.

This review article discusses aspects of hepatitis $C$ in healthcare workers and its risk of exposure and infection. Association between bloodborne pathogens and healthcare workers, hepatitis C (epidemiology, diagnosis, and prevention), communication and record of occupational injuries with biological material (blood and body fluids), and relationship between extension and consequences of accidents with cut and puncture objects and mucocutaneous exposure and the hepatitis $C$ were described.

Keywords Hepatitis C, Healthcare Workers, Occupational Injuries, Biological Material. 


\section{Introdução}

Mais de 10 anos se passaram desde que foram feitas as primeiras considerações sobre infecções adquiridas no trabalho pelos profissionais da saúde. A transmissão ocupacional a esses profissionais foi reconhecida em diversas doenças incluindo infecções causadas por microorganismos veiculados ao sangue como os vírus HIV (MARCUS et al., 1988), das hepatites B (MAST \& ALTER, 1993) e C (KIYOSAWA et al ., 1991; MITSUl et al., 1992; PURO et al., 1995) e Ebola (OUTBREAK OF EBOLA VIRAL HEMORRHAGIC FEVER - ZAIRE, 1995; ALTMAN, 1995), transmitidas por meio de contaminação oral e fecal como Salmonella (MISCHU et al., 1994) e vírus da hepatite A (HOFMANN et al., 1992; GERMANAUD et al., 1993) e contato direto como vírus do herpes (GRAVES et al., 1980) e Sarcoptes scabiei (VOSS \& WALLRAUCH, 1995; JIMENEZ-LUCHO et al., 1995).

A transmissão ocupacional é usualmente associada com a violação dos princípios básicos de controle da infecção, ou seja, lavagem das mãos e uso de equipamentos de proteção individual (EPI), vacinação aos profissionais da saúde e apropriado isolamento aos pacientes portadores de doença infecto-contagiosa.

A atenção sobre a transmissão dos patógenos relacionados ao sangue recebeu um impulso com a descoberta da síndrome da imunodeficiência adquirida e mais recentemente, do vírus Ebola. Embora, muitos profissionais que atuam em áreas não relacionadas à saúde estejam expostos a doenças causadas por organismos de transmissão (ar, oral ou fecal), os profissionais da saúde são um dos poucos grupos com risco de transmissão de patógenos relacionados ao sangue.

\section{Patógenos veiculados ao sangue e os profissi- onais da saúde}

A transmissão de patógenos veiculados ao sangue tem surgido como um importante risco ocupacional para os profissionais da saúde (PS). Informações sobre a transmissão destes patógenos para os PS têm sido obtidas através de vários estudos epidemiológicos individuais e em grupos. O conhecimento de como os patógenos são transmitidos e como a exposição ocorre são dados importantes para que se possam desenvolver estratégias preventivas.

Fatores determinantes do risco da transmissão ocupacional dos patógenos veiculados ao sangue são a prevalência da infecção na população, a probabilidade de se adquirir a infecção após um simples contato com o paciente infectado e a natureza e a freqüência do contato com o sangue. Estes fatores têm sido avaliados por estudos de soroprevalência, estudos prospectivos com PS após exposição a patógenos veiculados ao sangue e estudos de observação de contatos ocupacionais com sangue e outros fluidos corpóreos.

O fator de risco para a transmissão dos patógenos veiculados ao sangue é o resultado de uma exposição ocupacional onde são avaliados o paciente fonte (título do virus no sangue ou fluido corpóreo), a lesão (quantidade de sangue ou fluido corpóreo transferido para PS durante a exposição) e o profissional da saúde (estudo imunológico).

A discussão deste tema envolve o risco da transmissão ocupacional dos vírus da hepatites B (HBV), C (HCV) e o da imunodeficiência humana (HIV), que foi primeiramente descrita por McCORMICK e MAKI (1981). Desde então têm-se desenvolvido estudos da epidemiologia da exposição ao sangue, de estratégias de prevenção ao contato com o mesmo e das atitudes após a exposição (MARCUS et al., 1988; GERBERDING \& HENDERSON, 1992; LANPHEAR et al., 1994; GERBERDING, 1995; CDC, 1995).

Estudos em diferentes países têm demonstrado que os artigos pérfuro-cortantes são os materiais mais freqüentemente envolvidos nos acidentes com o PS, enquanto as causas mais comuns são: realização de sutura, recapeamento de agulha usando as duas mãos, instalação de dispositivos endovenosos, desconexão de agulhas da seringa, descarte de materiais pérfuro-cortantes em lugares inadequados ou em coletores cheios e agulhas expostas em lugares indevidos. ADEGBOYE et al. (1994) em um hospital universitário da Nigéria, identificaram 27\% de acidentes com agulha envolvendo 474 trabalhadores em relação aos acidentes com material biológico ocorridos. CAVALCANTE et al. (1991), em estudo sobre riscos de aquisição de HIV em profissionais de saúde, no Hospital Emílio Ribas em São Paulo, obser- 
varam que $50 \%$ dos acidentes eram causados por pérfuro-cortantes. Dados semelhantes foram demonstrados por MACHADO et al. (1992), em um hospital universitário de Ribeirão Preto, São Paulo. Na Itália, ALBERTONI et al. (1992), em um estudo multicêntrico, em 68 hospitais com 30.226 trabalhadores de saúde, mostraram que $29,3 \%$ sofreram acidente por agulha, no período de 1985 a 1990.

Acidentes envolvendo membrana mucosa podem ocorrer mediante respingos durante "intubação", aspiração de vias aéreas, ato cirúrgico ou algum outro procedimento de risco. O contato com pele lesada se faz, principalmente, durante procedimentos que envolvem grande quantidade de sangue. FAHEY et al. (1991), com o objetivo de analisar a freqüência de exposição não parenteral a sangue e fluidos corpóreos, em um Centro Clínico de Maryland (EUA), encontraram 60\% de exposição cutânea antes da implementação das precauções padrão e $51 \%$ após a implantação das mesmas. TOKARS et al. (1995), analisando 1.382 procedimentos cirúrgicos, descreveram a ocorrência de 1069 exposições com sangue na pele e 32 em membrana mucosa num estudo realizado com cirurgiões.

\section{Hepatite C}

As hepatites por vírus, em especial as denominadas $B$ e $C$, constituem um dos mais graves problemas de saúde médica e comunitária da população mundial, principalmente dos países em desenvolvimento. Apesar do grande progresso ocorrido a partir da década de 80 , especialmente no que se refere ao isolamento de novos vírus, determinação dos marcadores sorológicos, prevenção e terapêutica, ainda assim, as hepatites virais apresentam-se como importante agente etiológico da doença hepática aguda, insuficiência hepática e hepatopatias crônicas, ocorrendo sobretudo na população economicamente ativa, gerando grande prejuízo sob os aspectos sociais e econômicos.

É importante ressaltar a relevância do desenvolvimento da engenharia genética no final da década de 80 , permitindo a replicação e o armazenamento de clones ou frações de vírus. FEINSTONE et al. (1975), investigando 22 pacientes que tinham apresentado hepatite pós-transfusional com marcadores sorológicos das hepatites $A$ e $B$ negativos, definiram o termo não $A$ não $B$ (NANB) para esses casos. Assim, $\mathrm{CHOO}$ et al. (1989) conseguiram identificar, clonar e seqüenciar 0 genoma do vírus responsável pela hepatite NANB transmitida parenteralmente. Essa clonagem do vírus por meio da biologia molecular, permitiu o subseqüente desenvolvimento por KUO et al. (1989), do teste sorológico específico para a detecção dos anticorpos desencadeados pelo referido agente, que foi chamado vírus da hepatite C (VHC).

Apesar da dificuldade na visualização do VHC através da microscopia eletrônica, a clonagem molecular permitiv o conhecimento de sua organização genômica, incluindo-o nos RNA-vírus (MILLER \& PURCELL, 1990), da família flavivirídae, baseado na analogia com os conhecidos flavivírus, responsáveis pela dengue e febre amarela e os pestivírus, importantes patógenos animais, ambos pertencentes à referida família.

Até a recente descoberta do marcador sorológico da hepatite $C$, um porcentual significativo das doenças hepáticas crônicas, incluindo-se a cirrose, tinham a sua etiologia desconhecida, impossibilitando qualquer atuação preventiva e terapêutica específica. Em um estudo epidemiológico feito no Reino Unido (SAUNDERS et al., 1981), detectou-se que $35 \%$ dos pacientes cirróticos estudados entre 1959 e 1970 foram classificados como sendo doença hepática criptogênica. Estudos recentes (COURSAGET et al., 1990; JEFFERS et al., 1991), mostraram que a hepatite $C$ está freqüentemente envolvida nesses casos.

Atualmente é aceito que além do vírus $B, 0$ VHC também é importante fator predisponente ao carcinoma hepatocelular. Esta associação está bem documentada em trabalhos realizados em diferentes países (BRUIX et al., 1989; HASAN et al., 1990; COLOMBO et al., 1991 ;; SIMONETTI et al., 1992), mas principalmente no Japão (KIYOSAWA et al., 1990; NISHIOKA et al., 1991), onde já houve importante contribuição ao estudo da relação entre a hepatite $C$ e o hepatocarcinoma, dada a alta prevalência desta neoplasia naquele país.

\section{Epidemiologia}

A hepatite $C$ é considerada endêmica com 
distribuição universal. A prevalência na população ainda é pouco conhecida e uma variação genotípica é encontrada na distribuição geográfica (DIENSTAG et al., 1991; DIAS e WENDEL, 1997; FOCACCIA \& SOUZA, 1997; TENGAN, 1997).

A hepatite $C$ é atualmente um dos mais importantes problemas de saúde pública mundial, tendo prevalência de $3 \%(0,1$ a $5 \%)$ (INTERNATIONAL CONSENSUS CONFERENCE ON HEPATITIS C, 1999). A incidência de novos casos nos Estados Unidos é de 1 a 3 casos $/ 100$ mil/ano. Considerando que o HCV é assintomático na maioria dos casos, a infecção deverá ser a pandemia mais comum deste milênio. Atualmente, nesse país existem aproximadamente 2 a 3 milhões de pacientes com infecção crônica causada por esse vírus, cuja prevalência estimada é 1,8\% na população em geral e $0,5 \%$ em doadores de sangue (YONOSSI, 1997).

FOCCACIA, 1998, no Município de São Paulo, mostra que a prevalência estimada para HCV é de $1,4 \%$, sendo igual entre homens e mulheres. Maior prevalência por faixa etária ocorre a partir dos 30-39 anos $(2,2 \%)$ com aumento até $50-59$ anos $(3,8 \%)$. No Brasil, estudos sobre doadores de sangue têm mostrado prevalência entre 1,4\% e 3,1\% (TENGAN, 1997).

As vias de transmissão da HCV são parenteral, sexual, perinatal, por meio de contato domiciliar e ocupacional (ALTER, 1992; FOCACCIA \& SOUZA, 1997 ; TENGAN, 1997). A via parenteral é uma das formas mais importantes de transmissão da HCV, associada principalmente a transfusões de sangue e derivados e usuários de droga. Grupos de pacientes, como os hemofílicos e indivíduos em programas dialíiticos são considerados de alto risco, pela necessidade freqüente de sangue e derivados. Estudo de prevalência realizado por FORSETER (1993) em pacientes submetidos a hemodiálise detectou 19\% soropositivos para HCV.

A transmissão sexual dá-se por meio de contatos homossexuais e heterossexuais e está relacionada principalmente com a promiscuidade (múltiplos parceiros). A transmissão perinatal ainda não está bem esclarecida, não se sabendo se ocorre intra-útero por via transplacentária, durante o nascimento atra- vés do canal do parto, ou durante a amamentação (ALTER, 1992; TENGAN, 1997). O contato domiciliar e institucional, por sua vez, já foi relatado e continua entretanto pouco explicado. Sabe-se que contactantes domiciliares (não sexual) como filhos de pais portadores do HCV podem apresentar hepatite (ZUCKERMAN, 1995; DOEBELLING, 1997). O HCV tem sido também transmitido por doação de órgãos e a sorologia para HCV de doadores deve ser incluída como rotina no pré-transplante (PEREIRA, 1995; DAMANI \& KEYES, 1997).

A exposição ocupacional ao HCV é um sério problema para o trabalhador da área de saúde, já que a infecção subclínica é predominante e uma grande maioria dos portadores não têm sintomatologia na fase aguda. Além disso, os fatores de risco para transmissão ocupacional ainda são desconhecidos. O risco profissional inclui lesão por agulha, contato com pele não íntegra ou com membrana mucosa (DAMANI \& KEYES, 1997; DOEBELLING, 1997). A transmissão por mucosa ocular já foi documentada (SARTORI, 1993; ROSEN, 1997). Apesar do número de casos relatados de transmissão ocupacional ser pequeno, a transmissão por lesões percutâneas, no entanto, ainda representa a maior incidência.

Estudos retrospectivos no Japão mostram que o risco estimado da infecção pelo vírus HCV por lesão pérfuro-cortante está entre 27\% (KIYOSAWA et al., 1991) e 10\% (MITSUl et al., 1992). LANPHEAR et al., 1994 mostram uma taxa de soroconversão de $6 \%$ em um estudo realizado em Rochester, New York. Comparando, na Espanha um estudo com 81 PS que tinham sido lesados por materiais pérfuro-cortantes em contato com sangue de indivíduos hepatite $C$ positivo, não tinham evidências de contaminação com 1 ano de acompanhamento após a lesão (HERNANDEZ et al., 1992). Outro grupo de pesquisadores relata que o risco de contaminação se torna bastante alto quando a fonte está infectada com os vírus HIV e $\mathrm{HCV}$, embora estes resultados não sejam estatisticamente significantes (PURO et al., 1995).

A prevalência de $\mathrm{HCV}$ nos profissionais de saúde é variável. LANGE et al. (1995), em estudo realizado em Baltimore (EUA), encontraram 3\% dos indivíduos com marcadores 
positivos para anticorpos do HCV. GERBERDING (1994), realizando pesquisa no período de 8 anos (1984-1992), em São Francisco, mostrou prevalência de 1,4\% de anticorpos para HCV e ZUCKERMAN (1994), em Londres, encontrou 0,3\%. MARTINS (1996), avaliando 150 profissionais de saúde em Goiás, encontrou $4(2,6 \%)$ com anti-HCV positivo.

O risco de um indivíduo infectado pelo HCV transmitir o vírus em um acidente de trabatho pode estar relacionado com o tipo e o tamanho da amostra de sangue, como também pelo título de vírus que este apresenta, mas ainda há insuficiência de dados sobre qual a concentração do vírus necessária para transmitir a infecção. Além disso, não há padronização dos testes utilizados para medir esta infectividade, dificultando a avaliação do risco de transmissão das pessoas anti$\mathrm{HCV}$ recentes para outras.

\section{Diagnóstico}

O diagnóstico da hepatite $C$ se faz pela história clínica, dados epidemiológicos, exame físico, sorologia específica e exames complementares.

O diagnóstico etiológico é feito por intermédio da pesquisa de marcadores sorológicos como anti-HCV pelos método Enzyme-Linked Immunosorbent Assays (ELISA) ou Recombinat Immunoblot Assay (RIBA), ou ainda pela Polymerase Chain Reaction (PCR).

Atualmente, o NATIONAL INSTITUTE OF HEALTH (1997) recomenda na maioria dos casos o uso de testes ELISA de $2^{\underline{a}}$ e $3^{\underline{a}}$ geração; em casos de resultados negativos em pacientes de elevado risco, a utilização do teste confirmatório $P C R$, e em resultados positivos com resultado do PCR negativo, o teste RIBA.

\section{Prevenção}

Medidas preventivas para controle da hepatite $C$ devem ser tomadas principalmente em relação aos doadores de sangue.

A adoção de testes sorológicos na seleção de doadores de sangue diminuiv o risco de contágio pós transfusão. Testes sorológicos para prevenção de hepatite devem ser realizados em todos os componentes do sangue, podendo incluir pesquisa de anti-HCV por ELISA, RIBA e o PCR.

Apesar da redução na infecção póstransfusional devido a maior eficiência na seleção dos doadores de sangue, o número de indivíduos portadores tende a aumentar principalmente pelo elevado consumo de drogas injetáveis no mundo, ausência de fator de risco identificável na metade dos casos dificultando a prevenção e ocorrência de milhões de casos assintomáticos infectados nos últimos anos (INTERNATIONAL CONSENSUS CONFERENCEONHEPATITISC, 1999).

Medidas preventivas devem também ser adotadas em relação ao risco ocupacional em trabalhadores da área de saúde, com a medicina ocupacional participando ativamente realizando exames sorológicos na admissão, nos exames periódicos e sempre que houver acidentes com material biológico. Associar a estas ações a educação continuada, o uso de equipamentos de proteção individual e dispositivos que minimizem os riscos de acidentes de trabalho envolvendo sangue e outros fluidos corpóreos. Ainda não existe uma vacina ou imunoglogulina específica para HCV e o uso de imunoglobulina humana não é efetiva.

\section{Comunicação e registro de acidentes do trabalho}

Para poderem ser caracterizados na forma da lei, os acidentes do trabalho com materiais biológicos devem ser comunicados pelos acidentados e registrados pelas instituições empregadoras, havendo toda uma legislação específica sobre o assunto- Instituto Nacional do Seguro Social- Ordem de Serviço/INSS/DSS no 329/93 de 26/10/ 1993.

\section{Acidente do trabalho com materiais biológicos}

Os acidentes por contato com materiais biológicos humanos (sangue, secreções e excreções), através de acidentes do trabalho provocados pelo manuseio de objetos pérfuro-cortantes (agulhas, bisturis etc) e por 
respingos em mucosas, são acidentes do trabalho, podendo levar à doença profissional aguda, crônica ou até mesmo à morte.

Doenças infecto-contagiosas de longo período de incubação (síndrome de imunodeficiência humana, hepatites B e C, entre outras) podem ser transmitidas ao trabalhador por estes acidentes, necessitando controle sorológico periódico. A manifestação clínica e/ou sorológica dessas doenças, à luz dos conhecimentos científicos atuais, pode ocorrer até seis meses, em média, após o contágio.

\section{Extensão e conseqüências dos perfurocortantes $e$ acidentes mucocutâneos para Hepatite C}

Milhões de profissionais da área da saúde trabalham em hospitais ou outros estabelecimentos de saúde e estima-se que ocorram 600 mil a 800 mil acidentes perfurocortantes entre eles por ano, porém cerca da metade destes não são notificados. Na área hospitalar ocorrem aproximadamente 30 acidentes por 100 leitos por ano (EPINET, 1999).

A maioria dos acidentes envolve a equipe de enfermagem, porém, funcionários do laboratório, médicos, funcionários da limpeza e outros profissionais da saúde também se acidentam (McCORMICK \& MAKI, 1981; GERBERDING, 1995; CARDO, 1997; DOEBBELING, 1997).

No Brasil, CAVALCANTE et al. (1991) puderam observar que, dos 247 profissionais acidentados no Hospital Emílio Ribas de São Paulo, 57,5\% eram enfermeiras, 23,5\% médicos, 10,5\% pessoal de lavanderia e limpeza e $8,5 \%$ profissionais de laboratório. Ainda, MACHADO et al. (1992), em estudo realizado por 3 anos no Hospital das Clínicas da Faculdade de Medicina de Ribeirão Preto, demonstraram que, das categorias envolvidas, $55,5 \%$ eram auxiliares e técnicos de enfermagem, 19,4\% enfermeiros, $14,0 \%$ atendentes de enfermagem, $5,6 \%$ técnicos de laboratório, 2,8\% instrumentadores e 2,8\% escriturários.

O risco para a aquisição da hepatite $B$ pelo profissional da saúde é quatro vezes maior que o da população adulta em geral, que não atua em instituições de saúde (WEST, 1984). Quanto à hepatite C, o risco de aquisição deste patógeno pelo profissional da saúde é menor que o de adquirir HBV. Observa-se, entretanto, que o risco de aquisição do HCV pelos trabalhadores da área de saúde é maior que na população em geral, devido à transmissão deste se fazer principalmente por via parenteral (LEITE et al., 1992; SHORT \& BELL, 1993; ZUCKERMAN, 1994; PURO et al., 1995). POLISH et al. (1993), com o objetivo de estudar fatores de risco para hepatite $\mathrm{C}$ em profissionais de saúde, chegaram à conclusão que lesão por agulha é um dos fatores associados à infecção pelo HCV.

Alguns estudos têm demonstrado o risco da aquisição desses vírus pelos profissionais da saúde. PETROSILIO et al. (1995), analisando o soro obtido de 5.813 trabalhadores da saúde de cinco hospitais públicos na ltália, durante uma campanha de vacinação para HBV, observaram uma soroprevalência de $23,3 \%$ para $\mathrm{HBV}, 2 \%$ para $\mathrm{HCV}$. GERBERDING (1994), num estudo de 9 anos sobre a prevalência de HBV e HCV em 976 profissionais de saúde, encontrou inicialmente $21,7 \%$ e $1,4 \%$ respectivamente, excluindo os vacinados para HBV. Nesse mesmo estudo, seguindo 392 exposições percutâneas, documentou um caso de soroconversão para HCV em uma enfermeira, após acidente com agulha.

Quando o paciente-fonte é HCV positivo, o profissional da saúde tem alto risco de infecção pela hepatite C. Estudos de soroprevalência entre esses indivíduos mostraram 1 a $2 \%$ de anticorpo positivo (CDC, 1998; BELTRAMl et al., 2003). O acompanhamento desses profissionais, expostos a lesões percutâneas envolvendo sangue ou fluidos corpóreos com pacientes HCV positivo, mostrou que o risco de soroconversão é de aproximadamente 1,8\% (0 a 7\%). MITSUI et al. (1993), utilizando a PCR como método diagnóstico, constataram taxa de transmissão de $10 \%$.

Em casos de acidentes com profissionais da saúde envolvendo pacientes-fonte HCV positivo é importante informá-los sobre os riscos, uma vez que não existe vacina contra o vírus e o uso de imunoglobulinas nesses casos não parece ser eficaz (KRAWCZYNSKY 
et al., 1993).

Acompanhando os acidentes com material biológico notificados ( $n=436$ ) que ocorreram no Hospital de Base de São José do Rio Preto, SP, no período de janeiro de 1994 a dezembro de 1999, CIORLIA (2003) avaliou o seguimento sorológico realizado em profissionais da saúde com no mínimo 1 ano de acompanhamento após a exposição. Entre esses, não houve nenhum caso de soroconversão para hepatite $C$, que é rara como demonstrado por BALDO et al. (2002).

\section{Referências bibliográficas}

Adegboye AA, Moss GB, Soyinka F, Kreiss JK. The epidemiology of needlestick and sharp instrument accidents in Nigerian Hospital. Infect Control Hosp Epidemiol, Thorofare, 15(1):27-31, 1994.

Albertoni F, Ippolito $G$, Petinosillon N, Sommella L, Dinardo V, Ricci $C$ et al. Needlestick injury in hospital personnel: a multicenter survey from central Italy. Infect Control Hosp Epidemiol, Thorofare, 13(9):540-4, 1992.

Alter MJ, Margolis HS, Krawczynski K. The national history of community-acquired hepatitis $C$ in the United States. N Engl J Med, Waltham, 327:1899-905, 1992.

Altman LK. Deadly virus still spreads in Zaire: health workers are mainly affected. The New York Times May 12;Sec. A:6, 1995.

Baldo V, Floreani A, Dal Vecchio L, Cristofoletti M, Carletti M, Majori S, et al. Occupational risk of blood-borne viruses in healthcare workers: a 5-year surveillance program. Infect Control Hosp Epidemiol 2002;23:325-7.

Bruix J, Barrera JM, Calvet X, Ercilla G, Costa J, Sanchez-Tarias JM, et al. Prevalence of antibodies to hepatitis $C$ virus in Spanish patients with hepatocellular carcinoma and hepatic cirrhosis. Lancet, London, 8669:1004-6, 1989.
Além disso, nessa investigação a sorologia dos pacientes-fonte nos acidentes de trabatho notificados revelou $15,6 \%(n=71)$ de casos negativos, $63,4 \%(n=289)$ de desconhecidos e $21 \%$ ( $n=96)$ de casos positivos para vírus $\mathrm{HIV}, \mathrm{HBsAg}$ ou anti-HCV, sendo especificamente $34,4 \%(n=33)$ para HCV.

Efetiva profilaxia para o HCV é impossível de ser obtida até a presente data, mas esforços para reduzir a incidência das exposições ao sangue e fluido corpóreos devem ser incentivados.
Cardo DM. Patógenos veiculados pelo sangue. In: Rodrigues EAC et al. Infecções hospitalares: prevenção e controle. São Paulo: Sarvier; 1997. p.341-51.

Cavalcante NJF, Abreu ES, Fernandes ME, Richtmann R, Piovesana MN, Yamada FT. Risk of health care professionals acquiring HIV infection in Latin American. AIDS Care, Abingdon, 3(3):31 1-6, 1991.

CDC. Case-control study of HIV seroconversion in health-care workers after percutaneous exposure to HIV-infectedblood - France, United Kingdom and United States, January 1988-August 1994. MMWR, Atlanta, 44:92933,1995 .

CDC. Recommendations for prevention and control of hepatitis $\mathrm{C}$ virus (HCV) infection and HCV-related chronic disease. MMWR, Atlanta 47(No. RR-19): 1-33, 1998.

Ciorlia LAS. Acidentes de trabalho com material biológico e hepatites $\mathrm{B}$ e $\mathrm{C}$ entre profissionais da saúde ocupacionalmente expostos em um hospital universitário. Tese (Doutorado). Faculdade de Medicina de São José do Rio Preto. São José do Rio Preto, 2003.

Colombo M, Rumi MG, Donato MF, Tommasini MA, Del Ninno E, Ronchi $G$, et al. Hepatitis $C$ antibody in patients with 
chronic liver disease and hepatocellular carcinoma. Digest Dis Sci, New York, 36:1130-3, 1991.

Coursaget $P$, Bourdil C, Kastally R, Yvonnet $B$, Rampanarivo Z, Chiron JP, et al. Prevalence of hepatitis $C$ virus infection in Africa: anti-VHC antibodies in the general population and in patients suffering from cirrhosis or primary liver cancer. Res Virol, Paris, 141:449-54, 1990.

Choo QL, Kuo G, Weiner AJ, Overby LR, Bradley DW, Houghton M. Isolation of a DNA clone derived from a blood bovine non-A, non-B viral hepatitis genome. Science, Washington, 244:359-6, 1989.

Damani NN, Keyes JM. Hepatitis (B and C) and HIV infection. In: Northern Ireland, Craigavon Area Hospital Group Trust. Infection control: a procedure manual; 1997. p.1-17.

Dias MB, Wendel S. Infecções transmitidas pelo sangue. In: Rodrigues EAC et al. Infecções hospitalares: prevenção e controle. São Paulo: Sarvier; 1997. p. 304-31.

Dienstang JL, Wands JR, Isselbacher KJ. Acute hepatitis. In: Wilson JD et al. Harrison's principles of internal medicine. $12^{\text {th }}$ ed. New York: McGrow-Hill; 1991. p.132237.

Doebbeling BN. Protecting the health care workers from infection and injury. In: Wenzel RP. Prevention and control of nosocomial infections. $3^{\text {rd }}$ ed. Baltimore: Willians \& Wilkins; 1997. p.397-435.

EASL International Consensus Conference on Hepatitis C. J Hepathol, Amsterdam, 31(Supl 1):3-264, 1999.

EPINET [1999]. Needlestick prevention devices. Health Devices, Philadelphia, 28(10):381-407, 1999.

Fahey BJ, Koziol DE, Banks SM. Frequency of non parenteral occupational exposures to blood and body fluids before and after universal precautions training. Am J Med, New York, 90(2):145-53, 1991.

Focaccia R, Conceição OJG, Seth Jr H, Sabino E, Bassit L, Nitrini DR, et al. Estimated prevalence of viral hepatitis in the general population of the municipality of São Paulo, measured by a serologic survey of a stratified, randomized and residence-based population. Braz J Infect Dis, Salvador, 2(6):269-84, 1998.

Focaccia R, Souza FV de. Hepatites virais hepatite C. In: Veronesi R, Foccacia R. Tratado de infectologia. $9^{a}$ ed. São Paulo: Atheneu; 1997. p.314-22.

Forseter G, Wormser GP, Adler S, Lebovics E, Caimann M, O'Brien TA. Hepatitis C in the health care setting. II. Seroprevalence among hemodialysis staff and patients in suburban New York city. Am J Infect Control, Saint Louis, 21(1):5-8, 1993.

Gerberding JL. Management of occupational exposure to blood borne viruses. N Engl J Med, Waltham, 7(332):444-51, 1995.

Gerberding JL. Incidence and prevalence of human immunodeficiency virus, hepatitis $B$ virus, Hepatitis $C$, and cytomegalovirus among health care personnel at risk for blood exposure final report from a longitudinal study. J Infec Dis, Boston, 170:1410-7, 1994.

Germanaldi J, Causse X, Barthez JP. Prevalence of antibodies to hepatitis $A$ virus in health care workers [Letter]. Eur J Clin Microbiol Infect Dis, Wiesbaden, 12:5723, 1993.

Graves WL, Kaiser AB, Alford RH, Schaffner $\mathrm{WH}$. The problem of herpetic withtlow among hospital personnel. Infect Control, Thorofare, 1:381-5, 1980.

Hasan F, Jeffers $U$, Medina M, Reddy KR, Parker T, Schiff ER, et al. Hepatitis C associated hepatocellular carcinoma. Hepatology, Alexandria, 12;589-92, 1990. 
Hernandez ME, Broguera $M$, Puyuelo $T$, Barrera JM, Tapias JMS, Rodés J. Risk of needle-stick injuries in the transmission of hepatitis $C$ virus in hospital personnel. J Hepathol, Amsterdam, 16:56-8, 1992.

Hofmann F, Werhle G, Berthold H, Koster D. Hepatitis $A$ as an occupation hazard. Vaccine, Kidlinton, 10(Supl 1):82-4, 1992.

Jeffers $\amalg$, Hasan F, Medina M, Parker T, Schiff ER, Reddy KR. Prevalence of antibodies to hepatitis $C$ virus among patients with cryptogenic chronic hepatitis and cirrhosis. Hepatology, Alexandria, 15:187-90, 1991.

Jimenez-Lucho VE, Fallon F, Caputo C, Ramsey K. Role of prolonged surveillance in the erradication of nosocomial scabies in a extended care Veterans Affairs medical Center. Am J Infect Control, Saint Louis, 23:44-9, 1995.

Kiyosawa K, Sodeyama T, Tanaka E, Nakano Y, Furuta S, Nishioka K, et al. Hepatitis C in hospital employees with needlestick injuries. Ann Intern Med, Philadelphia, 115:367-9, 1991.

Kiyosawa K, Sodeyama T, Tanaka E, Nakano $Y$, Furuta S, Nishioka K, et al. Interrelationship of blood transfusion, non$A$, non- $B$ hepatitis and hepatocellular carcinoma: analysis by detection of antibody to hepatitis Cvirus. Hepatology, Alexandria, 12:671-5, 1990.

Kuo G, Choo QL, Alter HJ, Gitnick GL, Redeker AG, Durcell RH, et al. An assay for circulating antibodies to a major etiologic virus of human non- $A$, non- $B$ hepatitis. Science, Washington, 244:3624, 1989.

Lange WR, Frankenfield D, Weller E, Humphrey F, Mooney P, Bernacki EJ. Prevalence of hepatitis $B$, hepatitis $C$ and human immunodeficiency virus markers among hospital employment applicants. J Occup Environ Med, Baltimore, 37(4):486-9, 1995.
Lanphear BP, Linnemann Jr CC, Cannon CG, De Ronde MM, Pendy L, Kerley LM. Hepatitis $C$ virus infection in health care workers: risk of exposure and infection. Infect Control Hosp Epidemiol, Thorofare, 15(12):745-50, 1994.

Leite NC, Nogueira CM, Coeho HS, Perez R, Martins JJ, Soares JA, et al. Prevalência do anticorpo contra hepatite $\mathrm{C}$ (anti-HCV) em doadores de sangue do Rio de Janeiro, Brasil, sua relação com ALT e antiHBC+. Arq Gastroenterol, São Paulo, 29(1):5-11, 1992.

Machado AA. Risco de infecção pelo virus da imunodeficiência humana (HIV) em profissionais da saúde. Rev Saúde Publ, São Paulo, 26(1):54-6, 1992.

Marcus R. Suveillance of health care workers exposed to blood from patients infected with the human immunodeficiency virus. N Engl J Med, Waltham, 319:1118-23, 1988.

Martins RMB, Almeida VC, Vanderborght BOM, Brito JBA, Cardoso DDP, Pereira MS, et al. Prevalence of hepatitis $C$ antibodies among health care workers at high risk for blood exposure. Rev Inst Med Trop, São Paulo, 38(4):309-10, 1996.

Mast EE, Alter MJ. Prevention of hepatitis B virus infection among health care workers. In: Ellis RW, ed. Hepatitis B vaccines in clinical practice. New York: Marcel Dekker; 1993. p.295-307.

McCormick RD, Mak DG. Epidemiology of needle-stick injuries in hospital personnel. Am J Med, New York, 70:928-32, 1981.

Miller RH, Purcell RH. Hepatitis C virus shares aminoacid sequence similarity with pestiviruses and haviviruses as well as members of plant virus super groups. Proc Natl Acad Sci, Washington, 87:2057-61, 1990.

Mishu B, Koehler J, Lee JA, Rodrigue D, Brenner FH, Blake $P$, et al. Outbreak of Salmonella enterititis infection in the United 
States, 1985-1991. J Infect Dis, Chicago, 169-547-52, 1994.

Mitsui T, Iwano K, Masuko K, Yamasaki C Okamoto H, Tsuda F, et al. Hepatitis C virus infection in medical personnel after needlestick accident. Hepatology, Alexandria, 16:1109-14, 1992.

National Institute of Health. Consensus Development Conference Panel Statement Management of Hepatitis C. Hepatology, Alexandria, 26(Supl. 1):2-10, 1997.

Nishioka K, Watanabe J, Furuta S, Tanaka $\mathrm{E}$, lino $\mathrm{S}$, Suzuki $\mathrm{H}$, et al. A high prevalence of antibody to the hepatitis $C$ virus in patients with hepatocellular carcinoma in Japan. Cancer, Philadelphia, 67:429-33, 1991.

Outbreak of EBOLA viral hemorrhagic fever - Zaire, 1995. MMWR, Atlanta, 44:3812, 1995.

Pereira BJG. A controlled study of hepatitis $C$ transmission by organ transplantation. Lancet, London, 345:484-7, 1995.

Petrosillo N, Puro V, Jagger J, Ippolito G. The risks of occupacional exposure and infection by human immunodeficiency virus, hepatitis $B$ virus, and hepatitis $C$ virus in the dialysis setting. Italian Multicenter Study on Nosocomial and Occupacional Risk of Infections in Dialysis. Am J Infect Control, Saint Louis, 23:278-85, 1995.

Polish LB, Tong MJ, Co RL, Coleman PS, Alter MJ. Risk factor for hepatitis $C$ virus infection among health care personnel in a community hospital. Am J Infect Control, Saint Lovis, 21:196-200, 1993.

Puro V, Petrosillo N, Ipollito G. Risk of hepatitis $C$ seroconversion after occupational exposures in health care workers. Italian Study Group on Occupational Risk of HIV and Other Bloodborne Infections. Am J Infect Control, Saint Louis, 23:273-7, 1995.

Reed E, Daya MR, Jui J, Grellman K, Gerber $\mathrm{L}$, Loveless MO. Occupational infectious disease exposures in SEM personnel. J Emerg Med 11:9-16, 1993.
Rosen HR. Acquisition of hepatitis $C$ by a conjunctival splash. Am J Infect Control, Saint Louis, 25(3):242-7, 1997.

Sartori M, La Terra G, Aglietta M, Manzin A, Navino C, Verzetti G. Transmission of hepatitis $\mathrm{C}$ via blood splash into conjuntiva. Scand J Infect Dis, Stockholm, 25:270-1, 1993.

Saunders JB, Walter JRF, Davies P, Paton A. A 20-year prospective study of cirrhosis. Br Med J, Edinburgh, 282:262-6, 1981.

Short L, Bell DM. Risk of occupational infection with blood borne pathologens in operating and delivery room settings. Am J Infect Control, Saint Lovis, 6(21):34350, 1993.

Simonetti RG, Camma C, Fiorello F, Cottone $M$, Rapicetta M, Malino L, et al. Hepatitis $C$ virus infection as a risk factor for hepatocellular carcinoma in patients with cirrhosis. Ann Intern Med, Philadelphia, 116:97-102, 1992.

Tengan FM. Fatores de risco associados à infecção pelo virus da hepatite C [tese]. São Paulo (SP): Faculdade de Medicina da Universidade de São Paulo; 1997.

Tokars JI, Culver DH, Mendelson MH, Sloan $E P$, Farber BF, Fligner DJ, et al. Skin and mucous membrane contact with blood burring surgical procedures: risk and prevention. Infect Control Hosp Epidemiol, Thorofare, 16(12):703-11, 1995.

Voss A, Wallrauch C. Occupational scabies in health care worksers [Letter]. Infect Control Hosp Epidemiol, Thorofare, 16:4, 1995.

Younossi ZM. Chronic hepatitis C: a clinical overview. Cleve Clin J Med, Cleveland, 64:259-68, 1997.

Zuckerman AJ, Clewley G, Griffiths P, Cockcrof A. Prevalence of hepatitis C antibodies in clinical health care workers. Lancet, London, 343:1618-20, 1994.

Zuckerman AJ. Occupational exposure to hepatitis $B$ virus and human immunodeficiency virus: comparative risk analysis. Am J Infect Control, Saint Louis, 23(5):286-9, 1995. 